\title{
Reboxetina no tratamento da bulimia nervosa
}

\section{Reboxetine in the treatment of bulimia nervosa}

\author{
Simone Mancini Castilho e Luiz Henrique Viegas Costa ${ }^{\mathrm{b}}$ \\ anstituto de Psiquiatria da Faculdade de Medicina da Universidade de São Paulo. São Paulo, SP, Brasil. b Médico Psiquiatra. Clínica privada
}

Resumo É vasta a literatura demonstrando a eficácia dos antidepressivos inibidores seletivos da recaptação de serotonina na Bulimia Nervosa, diminuindo a frequiência do comportamento alimentar compulsivo e dos vômitos. A boa resposta terapêutica aos agentes farmacológicos noradrenérgicos, como a desipramina e a reboxetina, embora menos encontrada na literatura, também já foi documentada. $O$ presente relato de caso descreve o tratamento de uma paciente com Bulimia Nervosa utilizando-se reboxetina na dose de 4 a $8 \mathrm{mg}$ ao dia. A resposta terapêutica vem confirmar os resultados favoráveis do uso desta droga no tratamento da Bulimia Nervosa.

Descritores Bulimia. Bulimia nervosa. Antidepressivos. Reboxetina. Antidepressivos noradrenérgicos.

Abstract There is a substancial body of literature demonstrating the efficacy of selective serotonin reuptake inhibitors antidepressants (SSRI) in reducing binge eating and vomiting frequency in Bulimia Nervosa. Good therapeutic response to noradrenergic agents, like desipramine and reboxetine, though not frequently reported in literature, has already been demonstrated. This case report describes the treatment of Bulimia Nervosa with reboxetine (4 to $8 \mathrm{mg} /$ day) and its favorable therapeutic results.

Keywords Bulimia. Bulimia nervosa. Antidepressants. Reboxetine. Noradrenergic antidepressants.

\section{Introdução}

A causa multifatorial dos Transtornos Alimentares está bem estabelecida. O componente etiológico de alteração neuroquímica tem sido documentado em vários estudos. ${ }^{1,2}$ Os antidepressivos parecem exercer seus efeitos terapêuticos através da ação central em vias noradrenérgicas e serotoninérgicas. ${ }^{3,4}$ Entretanto, pode ser possível que a ação dos antidepressivos nos pacientes com Transtornos Alimentares seja monoaminérgica e decorra do restabelecimento da reduzida atividade noradrenérgica, como já foi proposto por alguns autores. ${ }^{3}$ A ação dos antidepressivos inibidores seletivos da recaptação de serotonina (ISRS), dentre eles a fluoxetina, na diminuição significativa, em relação ao placebo, do comportamento alimentar compulsivo e da freqüência de vômitos tem sido amplamente investigada e relatada na literatura. ${ }^{5,6}$ Embora os antidepressivos ISRS sejam recomendados como tratamento de primeira linha para a Bulimia Nervosa, estudos duplo-cegos controlados com desipramina mostraram superioridade dessa droga em rela- ção ao placebo, na redução da freqüência dos vômitos e episódios alimentares compulsivos. ${ }^{7}$ A resposta terapêutica de pacientes com Bulimia Nervosa a um agente farmacológico noradrenérgico como a desipramina, nos leva a supor que a reboxetina, um inibidor seletivo da recaptação de noradrenalina, também possa mostrar resultados terapêuticos positivos na Bulimia Nervosa, como aponta o estudo de EIGiamal et al. ${ }^{8} \mathrm{O}$ presente relato de caso confirma tais resultados favoráveis do uso de reboxetina no tratamento da Bulimia Nervosa.

\section{Apresentação do caso}

RMS, 29 anos, casada, superior completo, brasileira.

Desde a infância preocupava-se com sua aparência e forma física. Dos dez aos 17 anos lembra que fazia suas próprias dietas: só bebia líquidos ou ficava 24 horas sem se alimentar, comia apenas casca de pão e café e voltava a ficar em jejum; esporadicamente tomava, às escondidas, inibidores de apetite da sua mãe. Aos onze anos já fazia uso de laxantes, sempre 
preocupada com o peso. Com 17 anos iniciou dieta sob orientação de um endocrinologista e tomou hormônio tireoidiano, apresentando rápida e importante perda de peso. Passou, no entanto, a apresentar déficit cognitivo e claro prejuízo no desempenho escolar (duas reprovações consecutivas). Decidiu voltar a se alimentar, agora com 19 anos, porém, começou a apresentar episódios bulímicos nos quais quase diariamente, às escondidas e sem controle, ingeria alimentos diversos, doces e salgados simultaneamente, semipreparados e, às vezes, gelados. Algumas vezes tais episódios eram seguidos de vômitos auto-induzidos e, nessa ocasião, chegou ao seu peso mais alto: $62 \mathrm{Kg}$ e Índice de Massa Corpórea de $25 \mathrm{Kg} / \mathrm{m}^{2}$. Desde então nunca apresentou remissão completa da sintomatologia. Atualmente pesa $54 \mathrm{Kg}$, mede 1,59 m, apresenta Índice de Massa Corpórea de 21 e se acha gorda. Pesa-se semanalmente, sempre após ir ao banheiro e imediatamente antes do almoço. Tem sérios descontroles alimentares e vomita quase diariamente. Agride-se fisicamente quando acha que comeu demais ("socos na barriga"), culpa-se, sente raiva de si. Apresenta irregularidade menstrual e sintomas depressivos associados: desânimo, tristeza, hipersônia, irritabilidade, baixa auto-estima, tensão e dores musculares. Sempre bastante introspectiva, nunca contou a ninguém sobre seus problemas alimentares, nem mesmo à psicoterapeuta com quem tentou se tratar há algum tempo.

Em meados de 2000 a paciente, que nunca tinha tomado antidepressivo, procurou um psiquiatra com queixas depressivas e iniciou tratamento com $75 \mathrm{mg}$ de venlafaxina ao dia. Apresentou melhora parcial dos sintomas depressivos nas primeiras semanas, mas agravamento dos episódios bulímicos e ganho de peso, o que a levou a relatar o problema alimentar ao psiquiatra. Em dezembro de 2000, iniciou uso de $4 \mathrm{mg}$ diários de reboxetina, com boa resposta terapêutica, melhora das compulsões alimentares (uma ou duas vezes por semana) e nítida diminuição na freqüência dos vômitos. Também melhorou dos sintomas depressivos; sentia-se bem e decidiu, nesta ocasião, marcar a data de seu casamento. A obstipação intestinal foi o único efeito colateral apresentado. Fazia terapia, mas jamais relatara o Transtorno Alimentar para seu terapeuta. Dessa forma, não foi realizada nenhuma intervenção psicoterápica dirigida ao problema alimentar ou a qualquer outro tipo de orientação que não a clínica. Sua melhora manteve-se até meados de 2001, quando apresentou retorno da compulsão alimentar, às vésperas de seu casamento. Foi feito ajuste na dosagem da medicação para $8 \mathrm{mg}$ de reboxetina ao dia, com boa resposta. Manteve-se bem por quase um ano, fazendo uso concomitante de reeducador intestinal. Com a suspensão da medicação por iniciativa própria, após estar se alimentando de forma apropriada, sem restrições, não vomitando e estar gostando de sua forma física, voltou a piorar dos sintomas alimentares e depressivos que remitiram com a reintrodução da medicação, na dose de $4 \mathrm{mg}$ por dia. Atualmente a paciente passa bem, estável e assintomática; não apresenta compulsões alimentares, vômitos, uso de outros métodos purgativos e sua imagem corporal está preservada.

\section{Discussão}

O presente relato de caso nos confronta com a excelente resposta terapêutica à reboxetina apresentada pela paciente em questão, embora seja curto o período de seguimento. Vale ressaltar, ainda, que a maioria dos pacientes não tem uma resposta tão eficaz à medicação, usada de forma isolada (sem a associação, por exemplo, da terapia cognitivo-comportamental), sendo comum permanecerem algumas distorções e atitudes quanto ao peso e forma corporal. Além disso, o uso de antidepressivos na Bulimia Nervosa geralmente leva a uma resposta melhor no início do tratamento, com redução da freqüência de compulsões alimentares e dos métodos purgativos, que podem não se manter com o passar do tempo.

Nesse caso, a nítida piora do quadro alimentar após a interrupção da medicação (com melhora após sua reintrodução) e o fato de não ter havido outras intervenções para os sintomas alimentares que não a farmacológica, aumentam a chance da resposta terapêutica poder ser atribuída à medicação.

Visto que, no caso apresentado, os sintomas depressivos e alimentares melhoraram concomitantemente, após a introdução da reboxetina, fica difícil descartar a possibilidade da diminuição dos sintomas alimentares ser conseqüente à melhora dos sintomas depressivos. Por outro lado, o fato da paciente ter melhorado de vários sintomas depressivos com o uso de venlafaxina e ter apresentado piora dos sintomas alimentares, torna a hipótese apresentada menos provável e reforça a idéia de uma ação específica da reboxetina nos sintomas alimentares. Entretanto, cabe notar que a venlafaxina pode não ter auxiliado na diminuição dos sintomas alimentares por ter sido usada apenas na dose de $75 \mathrm{mg}$ ao dia e não em doses mais elevadas, onde se conhece sua ação noradrenérgica. ${ }^{8}$ Além disso, a presença de náuseas, efeito colateral possível quando do uso de venlafaxina, pode manter e agravar o ciclo compulsão-vômitos nesses pacientes, piorando o quadro clínico.

Encontramos na literatura apenas um artigo relatando o tratamento de sete casos de Bulimia Nervosa com reboxetina. Os achados mostram redução significativa na freqüência de episódios bulímicos e no uso de métodos purgativos pelas pacientes. ${ }^{8}$ Não há trabalhos com grupo controle ou número maior de pacientes para que pudéssemos atribuir, de forma mais segura, os resultados positivos, à droga em questão.

De qualquer forma há a possibilidade de haver alguma alteração na modulação noradrenérgica do comportamento alimentar nos pacientes com Bulimia Nervosa, como já proposto por Kaye et al. ${ }^{10}$ Relatos de reduzida atividade noradrenérgica no sistema nervoso central e periférico de pacientes com transtornos alimentares sob condições diferentes de estimulação (basal e stress), também são encontrados. ${ }^{11}$ Além disso há evidência de que os inibidores seletivos da recaptação de noradrenalina produzem uma diminuição da ingesta alimentar em modelos animais. ${ }^{12}$

Apesar dos antidepressivos inibidores seletivos da recaptação de serotonina (ISRS) serem o tratamento farmacológico de primeira linha para a Bulimia Nervosa, a imipramina e a desipramina foram as primeiras substâncias 
a serem examinadas nesse grupo de pacientes. ${ }^{13}$ A desipramina é um antidepressivo tricíclico amina secundária com afinidade pelos sítios de recaptação da noradrenalina. Em estudos controlados duplo-cegos, de avaliação da desipramina, a redução na freqüência de episódios bulímicos variou de $40 \%$ a $90 \% .^{14-16}$

$\mathrm{O}$ antidepressivo tricíclico desipramina apresenta propriedades farmacodinâmicas não relacionadas à noradrenalina, que são responsáveis por alguns efeitos colaterais difíceis de serem tolerados, especialmente no tratamento em longo prazo. Há, dessa forma, altas taxas de abandono nos tratamentos com essa droga. ${ }^{7} \mathrm{Um}$ antidepressivo como a reboxetina, de perfil noradrenérgico, seletivo, com pouca ou nenhuma ação em receptores comumente envolvidos no surgimento de efeitos co-

\section{Referência}

1. Fava M, Copeland P, Schfweiger U, Herzog, D. Neurochemical abnormalities of anorexia nervosa and bulimia nervosa. Am J Psychiatry 1989;146:963-71.

2. Levy B. Neuroendocrine profile in bulimia nervosa. Biol Psychiatry 1989;25:98-109.

3. Pirke K, Platte P, Laessle R, Seidl M, Fichter M. The effect of a mental challenge test of plasma norepinephrine and cortisol in bulimia nervosa and in controls. Biol Psychiatry 1992;32:202-6.

4. Leibowitz S. The role of serotonin in eating disorders. Drugs 1990;39(Suppl 3):22-48.

5. Fluoxetine Bulimia Nervosa Collaborate Study Group. Fluoxetine in the treatment of bulimia nervosa. Arch Gen Psychiatry 1992;49:139-47.

6. Goldstein D, Wilson M, Thompson V, Potvin J, Rampey A. For the Fluoxetine Bulimia Nervosa Research Group. Long-term fluoxetine treatment of bulimia nervosa. Br J Psychiatry 1995;166:660-6.

7. Walsh T, Hadigan C, Devlin M, Gladis M, Roose S. Long-term outcome of antidepressant treatment for bulimia nervosa. Am J Psychiatry 1991;148:1206-12.

8. El-Giamal N, Zwaan M, Bailer U, Lennkh C, Schüssler P, Strnad A, et al. Reboxetine in the treatment of bulimia nervosa: a report of seven cases. Int Clin Psychopharmacol 2000;15:351-6.

9. Malhotra S, King K, Welge J, Brusman-Lovins L, McElroy S. Venlafaxine treatment of binge-eating disorder associated with obesity: a series of 35 patients. J Clin Psychiatry 2002;63(9):802-6.

10. Kaye W, Ballenger J, Lydiard B, Stuart G, Laraia M, O Neil P, et al. CSF monoamine levels in normal weight bulimia: evidence for abnormal noradrenergic activity. Am J Psychiatry 1990;147:225-9. laterais, pode representar uma opção favorável em pacientes com Bulimia Nervosa.

\section{Conclusão}

Apesar da falta de dados para uma afirmação contundente, parece que a reboxetina pode ser um antidepressivo eficaz no tratamento da Bulimia Nervosa, com grande efeito na diminuição da freqüência dos episódios alimentares compulsivos e da prática de métodos purgativos. Podendo tornar-se, assim, uma alternativa farmacológica a ser utilizada no tratamento dessa patologia.

A fim de que tais impressões possam se confirmar, estudos duplo-cegos controlados com placebo utilizando reboxetina, são necessários e de grande interesse científico.

11. Pirke K M. Central and peripheral noradrenalin regulation in eating disorders. Psychiatry Res 1996;62:43-9.

12. Gehlert D, Dreshfeld L, Tinsley F, Benvenga M, Gleason S, Fuller R, et al. The selective norepinephrine reuptake inhibitor, LY368975, reduces comsuption in animal models of feeding. JPET 1998;287:122-7.

13. Agras W, Dorian B, Kirkley B. Imipramine in the treatment of bulimia: a double-blind controlled study. Int J Eating Disord 1987;6:29-38.

14. Barlow J, Blouin J, Blouin A, Perez E. Treatment of bulimia with desipramine: a double-blind crossover study. Can J Psychiatry 1988;33:129-33.

15. Blouin A, Blouin J, Perz E, Bushnik T, Zuro C, Mulder E. Treatment of bulimia with fenfluramine and desipramine. J Clin Psychopharmacol 1988;8:261-9.

16. Hughes P, Wells L, Cunningham C, Ilstrup D. Treating bulimia with desipramine. Arch Gen Psychiatry 1986;43:182-6.

\section{Correspondência:}

Simone Mancini Castilho

Rua Lírio do Amazonas, 20 Jardim Haras Bela Vista

06730-000 Vargem Grande Paulista, SP, Brasil

Tel: (0xx11) 4158-2035/ 9337-4651

E-mail: simonemcastilho@hotmail.com 